\title{
Conferences in the Field of Chemical Sciences Held in 2013
}

July 28-August 1

Fort Collins, USA

July 28-August 2

Taipei, Taiwan

August 4-9

Sapporo, Japan

August 11-16

Istanbul, Turkey

August 13-16

Greenville, USA

August 18-22

Durham, UK

August 19-23

Sentosa, Singapore

August 25-28

Yerevan, Armenia

August 25-29

Warsaw, Poland

September 1-5

Sopron, Hungary

September 1-6

Prague, Czech Republic

September 6-9

Golden Sands, Bulgaria

September 22-25

Frankfurt, Germany

September 22-27

Budapest, Hungary

October $21-24$

Nice, France

October 27-31

Brisbane, Australia

December 15-19

New Delhi, India 17th IUPAC International Symposium on Organometallic Chemistry Directed Towards Organic Synthesis (OMCOS 17)

http://www.omcos17.com/

15th International Symposium on Novel Aromatic Compounds (ISNA-15) http://www.isna15.org/

16th International Symposium on Relations between Homogeneous and Heterogeneous Catalysis (ISHHC-16)

http://www.shokubai.org/ishhc16/

44th IUPAC Congress - Clean Energy Through Chemistry

http://iupac2013istanbul.org/

15th International Symposium on MacroMolecular Complexes (MMC-15)

http://macromolecularcomplexes.org/index.html

10th International Conference on Advanced Polymers via

Macromolecular Engineering (APME-2013)

http://www.dur.ac.uk/soft.matter/apme2013

15th Asian Chemical Congress

http://15acc.org/

Frontiers in Chemistry, Armenia (ArmChemFront 2013)

http://www.armchemfront.com/

XVIIth European Conference on Analytical Chemistry (EuroAnalysis XVII) http://www.euroanalysis2013.pl/

9th European Conference of Computational Chemistry (EuCo-CC)

http://www.euco-cc9.mke.org.hu/home.html

14th European Symposium on Organic Reactivity (ESOR 2013)

http://www.icaris.cz/conf/esor2013/?id=343\&lng $=$ cz

5th International Symposium on Advanced Micro- and Mesoporous Materials http://micro2013.innoslab.com/

1st European Conference on Natural Products

http://events.dechema.de/events/en/Events/1st+European+Conference+ on + Natural+Products.html

10th Applied Geochemistry Conference

http://www.aig10.com/

International Polysaccharide Conference

http://epnoe2013.sciencesconf.org/

5th Asia-Pacific NMR Symposium (APNMR5)

http://apnmr2013.org/

2nd Asian Congress on Biotechnology (ACB2013)

http://web.iitd.ac.in/ sundar/acb2013.htm 